\title{
LA EXPERIENCIA COMO CATEGORÍA DE ANÁLISIS DE LA RECREACIÓN: POSIBILIDAD PEDAGÓGICA
}

\author{
EXPERIENCE AS ANALYSIS CATEGORY OF RECREATION: A PEDAGOGICAL POSSIBILITY
}

Resumen

El artículo de reflexión, derivado de la investigación "Pedagogía y metodología de la recreación en la escuela"1 ${ }^{\prime \prime}$ presenta una propuesta sobre cómo la experiencia se constituye en una categoría de trabajo o análisis de la recreación. Se retoman aportes teóricos de autores como Jhon Dewey, Csikszentmihslyi, Glasersfeld y Kelly, entre otros, para configurar el marco explicativo de la experiencia y su relación con la pedagogía desde la recreación.

Palabras clave: experiencia, pedagogía, educación, escuela.

Abstract

This reflection paper is derived from the research "Pedagogy and Methodology for School Recreation". It presents a proposal of how experience is constituted as an analysis category of recreation. Theoretical contributions from authors such as John Dewey, Csikszentmihslyi, Glasersfeld and Kelly, among others, are taken into account to set the explanatory framework for the experience concept and its connection to pedagogy from recreation.

Keywords: experience, pedagogy, education, school.

Fecha de recepción: 12 de diciembre de 2013

Fecha de aprobación: 17 de marzo de 2014

\footnotetext{
* Magíster en Educación. Psicólogo. Docente e investigador de la Licenciatura en Recreación Universidad Pedagógica Nacional. Correo electrónico: piliox@hotmail.com

** Magíster en Investigación Social Interdisciplinaria, Especialista en Investigación Social y Licenciado en Educación Física. Docente e investigador de la Licenciatura en Recreación, Universidad Pedagógica Nacional. Correo electrónico: juanmacc@yahoo.com

*** Magíster en Educación, Licenciada en Educación Física. Docente e investigadora de la Licenciatura en Recreación, Universidad Pedagógica Nacional. Coordinadora del mismo programa. Correo electrónico: astridbibianarc@yahoo.com

1 Proyecto de investigación del grupo Lúdica, Cuerpo y Sociedad, de la Licenciatura en Recreación, Facultad de Educación Física, Universidad Pedagógica Nacional, financiado por el Centro de Investigaciones de la Universidad Pedagógica Nacional (CIUP). Código FEF 359-13. Investigadora principal: Astrid Bibiana Rodríguez Cortés.
} 


\section{ANCLAJES TEÓRICOS SOBRE EXPERIENCIA}

\section{La postura de Dewey}

Una primera aproximación al concepto de experiencia la realiza Dewey en su libro Experiencia y educación (1945), el autor la describe bajo dos premisas, la experiencia que actúa como medio de significación del sujeto y la experiencia que posee un carácter transitorio.

El primer enfoque está orientado a entender las experiencias como aquellas que organizan la vida mental y emocional de los sujetos guiándolas hacia fines posteriores, estas se autoorganizan para dar vida a otras experiencias aumentando la complejidad en la forma como el sujeto vivencia su relación con el entorno y las posibilidades de sus acciones mentales, emocionales y sociales; este tipo de experiencia posee un horizonte que permite la elaboración de condiciones apropiadas para el sujeto en el sentido de generar un sistema a través del cual la experiencia encadena las motivaciones al dar cuenta de la realidad en la cual interactúa. En este sentido, Dewey asigna un valor central a cómo las experiencias pueden ser organizadas sistemáticamente para hacer de ellas mediaciones que orienten a los individuos hacia la autonomía. El logro de este tipo de acción es generado por los procesos educativos que deben remplazar las experiencias pedagógicas, de modo que los conocimientos aprendidos estén conectados con situaciones intencionales del yo. Dewey (1945) considera que la experiencia en lo educativo se debe organizar por métodos para favorecer de forma auténtica las vivencias, asimismo, evidencia la necesidad de orientar una experiencia organizada al crecimiento del individuo en la escuela; en otras palabras, la experiencia debe favorecer el enriquecimiento de la comprensión del sujeto sobre el mundo con el que interactúa validada en cierto sentido desde lo pedagógico e instaurada por elementos favorables de la cultura para buscar en el individuo una vida mental que le permita reconstruir el contexto en el cual interviene.

A su vez, Dewey (1945) plantea dos criterios para identificar cuando una experiencia es significativa para el sujeto; el primero de ellos es la continuidad experiencial, que hace alusión al hábito como medio por el cual se mantiene en el tiempo una determinada experiencia como anclaje de otra, en relación con ello, expresa:

La característica básica del hábito es que toda experiencia emprendida y sufrida modifica al que actúa y sufre, afectando esta modificación, lo deseemos o no, a la cualidad de las experiencias siguientes, pues quien interviene en ellas es una persona diferente (p. 34).

Este principio permite comprender la experiencia como una construcción y reconstrucción de la vida de un individuo en la medida que una nueva información, conocimientos o vivencias se articulan en torno a mapas elaborados en la interacción pasada con una determinada situación. El sustento no es otra cosa que el hábito construido como esquema a través del cual la experiencia se manifiesta como constitución de una realidad mental. De igual modo, es posible la modificación continua del hábito debido a la dialéctica que se genera con las nuevas construcciones mentales, según Dewey (1945), "el principio de continuidad de la experiencia significa que toda experiencia recoge algo de la que ha pasado antes y modifica en algún modo la cualidad de la que viene después" (p. 34). Sin embargo, el autor en mención hace la salvedad que el principio de continuidad puede en ocasiones presentarse para mantener ciertos tipos de hábitos que generan actitudes no favorables para el sujeto, la continuidad puede operar en dirección contraria y bloquear el desarrollo o crecimiento de un sujeto a la integración como ciudadano, de ahí la pertinencia de orientar desde lo educativo la experiencia del sujeto.

Siguiendo la línea de argumentación anterior, Dewey aduce que una experiencia significativa debería producir curiosidad, movimiento, deseo de ir en una determinada dirección por procesos de autorregulación y autonomía, sin necesidad de referencias externas que alienten un modelo heterónomo, ser con uno, los otros y el contexto, esto involucra la coexistencia de un contrato social. Teniendo en cuenta esto, una de las funciones de lo educativo o del educador es establecer hacia dónde se dirige la experiencia del estudiante y orientarla para que sea lo más beneficiosa posible. Por último, como condición de este criterio (continuidad experiencial), Dewey considera que la experiencia posee un carácter activo, no es solo una construcción que realiza el sujeto de forma interna, sino que son necesarias condiciones externas al sujeto para darse de forma significativa; esta condición de lo externo coloca a la experiencia en el terreno de las relaciones sociales como vehículo de facilitación de la construcción de la experiencia, inclusive la construcción sociocultural parte del hábito de 
las experiencias acumuladas históricamente y continuamente reelaboradas para dar respuesta a las necesidades de la sociedad en la que se vive, en este sentido, Dewey (1945) expresa:

Toda experiencia auténtica tiene un aspecto activo que cambia en algún grado las condiciones objetivas bajo las cuales se ha tenido la experiencia (...) En una palabra, vivimos del nacimiento a la muerte, en un mundo de personas y cosas, que en gran medida es lo que es por lo que han hecho y transmitido las actividades humanas anteriores (p. 40).

El segundo criterio para identificar cuándo una experiencia es valiosa es la interacción. Para introducir está concepción, Dewey plantea que la experiencia posee dos características constitutivas: las objetivas y las internas. Juntas constituyen lo que él denomina una situación. En el ámbito educativo, se le ha dado relevancia a las características objetivas, aquellas fuera del sujeto, diseñadas por agentes independientes de quien las vivencia; las internas se han negado por considerar que, al pensarse condiciones para otros, por sí solo, lo objetivo desarrollará, adaptará o ampliará la interna.

Ahora bien, la interacción se da dentro de unas redes sociales de significados establecidas por un conjunto de situaciones donde el individuo interactúa de modo intencional y configura sus experiencias como parte de su identidad, dicha interacción se puede dar con herramientas o símbolos culturales; un ejemplo es el niño que juega con un carro de juguete, él está inmerso en una situación en la que el carro representa un símbolo con el cual se interactúa, se construye un modo de acción social y refleja el mundo donde se vive, no obstante, para que el carro de juguete se exprese como una experiencia significativa, la acción sobre él debe mantener una relación de continuidad experiencial en el tiempo y representar una realidad sujeta de ser experimentada por el niño, así como convertir la acción mental del niño en interacción con el entorno, según Dewey (1945):

Una experiencia es siempre lo que es porque tiene lugar una transacción entre el individuo y lo que, en el momento, constituye su ambiente, y si este último consiste en personas con las que se está hablando sobre algún punto o suceso, el objeto sobre el que se habla forma parte de la situación (p. 46).
La interacción se configura como parte de la experiencia del individuo y adquiere sentido dentro de un contexto determinado y sobre ella se reconfigura cada vez que la situación esté presente, logrando con ello un aprendizaje y estados motivacionales que lo permitan.

En otro sentido, la experiencia significativa con sus dos criterios (continuidad e interacción) logra configurar un sistema por medio del cual el sujeto apropia hábitos del entorno a sus propios hábitos, guiando la experiencia como vehículo de aprendizaje; la experiencia solo tendría sentido en la medida que se pueda asociar con experiencias significativas previas en consonancia con aspectos motivacionales, pasiones y deseos construidos en la misma experiencia. Si un individuo se acerca por primera vez a una situación y su interacción no le permite asociar significando con experiencias previas o no pertenece a una acción que consienta dimensionar sus elementos prácticos con lo cotidiano, la situación, a pesar de generar una experiencia, anula la posibilidad de la continuidad experiencial; determina una pauta de no conexión entre experiencias. Para Dewey (1945), "La actitud más importante que se puede formar es la de desear seguir aprendiendo" (p. 53).

Siguiendo con lo anterior, Dewey, en el libro Democracia y Educación (2004), hace la distinción sobre la naturaleza de la experiencia en el sentido de especificar los pasos que sigue la experiencia en el sujeto; inicialmente, toda experiencia es activa, o sea, parte de la realización de actividades, de interacciones con el entorno, de ensayar. Por otro lado, la experiencia es pasiva, es lenta, requiere de tiempo y de sufrimiento, pues si la actividad generada en la experiencia modifica o interviene en el acto cognoscitivo del sujeto, modifica sus estructuras de relación y genera aprendizaje, se mueve a una acción que se reflexionó y produjo emergencia de anticipación de la experiencia. Esto representa dos fases de la experiencia, la primera es aquella que la configura, un evento, una situación, una vivencia, algo que le pasa al sujeto, permitiéndole desarrollar un tipo de movimiento intencional; la segunda es la que convierte la acción en reflexión, en posibilidad mental trascendente en el tiempo y que el sujeto estima conveniente asumir como parte de su relación con el contexto. Según Dewey (2004): 
1) La experiencia es primariamente un asunto activo-pasivo; no es primariamente cognoscitiva. 2) Pero la medida del valor de una experiencia se halla en la percepción de las relaciones o continuidades a que conduce. Comprende conocimiento en el grado que se acumula o se suma algo o tiene sentido (p. 125).

El segundo tipo de experiencia para Dewey es la transitoria, que hace referencia a circunstancias cotidianas a las cuales se ven expuestos todos los sujetos, pero no constituyen acciones que enriquezcan la vida mental o que contribuyan a la estabilidad del sujeto como agente autónomo, tender una cama o cepillarse los dientes son ejemplo de ellas, es la experiencia automática asentada en automatismos o en situaciones carentes de motivación para el sujeto.

\section{El aporte de Glasersfeld}

La experiencia puede ser considerada como la forma en que el conocimiento se va adaptando a condiciones del medio y al desarrollo mental de los sujetos, la experiencia solo se constituye como tal cuando se puede dar cuenta de ella a través de representaciones mentales y expresadas hacia fuera por medio de símbolos. Sin estas dos condiciones, la experiencia no posee trascendencia humana más allá de la establecida por las condiciones orgánicas de cualquier especie. Por tanto, la experiencia tiene una función adaptativa-transformadora, siendo esta de carácter cognitivo, en la medida de permitir dar cuenta de ella misma. Glasersfeld (1996), al retomar a Piaget, considera: "Piaget ha reiterado innumerables veces que, desde su perspectiva, la cognición debe ser considerada una función adaptativa" (p. 31), esto último lleva a considerar que el conocimiento se da en la relación con el mundo sociocultural y otros sujetos, según Glasersfeld, "la experiencia de un ser humano siempre incluye la interacción social con otros sujetos cognoscentes" (p. 33).

En la anterior dirección, la experiencia tendría por lo menos tres componentes y configurar un esquema de acción: 1) reconocimiento de un evento o situación (implica una experiencia anterior, o un aprendizaje), 2) una asociación del evento o situación con un antecedente similar que permita clasificarlo y darle sentido y 3) una expectativa sobre el evento o situación que permita anticipar, recrear opciones sobre ella o dirigir al sujeto a la búsqueda de situaciones de recompensa sobre la misma experiencia que permita mantenerla en el tiempo. Cuando en el tercer componente la experiencia no genera expectativa, esta cae en un terreno donde entra en conflicto con los eventos o situaciones anteriores, creando una bifurcación para la generación de nuevas experiencias y aprendizajes, con ello se da paso a lo denominado como conflicto de experiencia 0 , en términos psicológicos, a un conflicto cognitivo entre los antecedentes de la experiencia y la experiencia presente. A su vez, la experiencia requiere del sujeto las siguientes capacidades:

- Representar las experiencias, para ello es necesario recordar y recuperar información significativa.

- Realizar contrastación de información a través de comparaciones, clasificaciones y emisión de juicios.

- Mantener continuidad en el flujo de la experiencia.

- Buscar determinado grupo de experiencias por intención propia, lo que implica tener heurísti$\cos ^{2}$ de búsqueda generados en la misma construcción de la experiencia.

Esta postura de Glasersfeld propone un modelo de la experiencia humana sustentada sobre la interacción social basada en el intercambio de representaciones a través del lenguaje. Toda experiencia lo es en la medida que su carga de significados puede ser expresada a otros por medio de un sistema de mutuas comprensiones mentales basadas en regularidades sociales. Por tanto, la experiencia se convierte en un proceso de construcción sobre la realidad que se da en comunidad.

\section{Lo óptimo desde Csikszentmihslyi y Csikszentmihslyi}

Csikszentmihslyi y Csikszentmihslyi (1998) introducen el concepto de experiencia de flujo para explicar lo sucedido con los individuos sumergidos en determinadas tareas, dejándose llevar por ellas hasta su conclusión, pero una vez finalizadas perdían el interés por lo realizado, abandonando o desechando el producto elaborado. Las explicaciones sobre la recompensa obtenida después de realizado un determinado trabajo, como dinero, reconocimiento, el deber cumplido, no eran suficientes para dar cuenta de lo acaecido en los sujetos durante el proceso de elaboración del producto, pues ninguna recompensa justifica la inversión de tiempo y esfuerzo realizada. El autor considera que

2 Se entiende por heurístico, según la Real Academia de la Lengua Española, "técnica de la indagación y del descubrimiento". 
las personas se vinculan a procesos cuando la motivación intrínseca es la que determina el horizonte de la experiencia, cuando el sujeto establece la dirección en la cual desea invertir sus esfuerzos, dejando de lado los intereses externos como regulación de las acciones; normalmente se considera que los sujetos orientan sus pensamientos y acciones debido a las recompensas que puede obtener del medio al realizar una determinada tarea y que ello se mantiene en el tiempo permitiendo esquemas permanentes de acción y de conductas; el autor determina que esto no es cierto en buen número de casos, pues los sujetos pueden establecer patrones internos lejanos de las regulaciones externas en la medida que sienten una vinculación especial con lo que hacen, lo disfrutan y se convierte en un sentido de implicación sobre la vida que supera las condiciones de las reglas sociales.

El autor considera que la experiencia de flujo se relaciona con la búsqueda de acciones que tienen fin en sí mismo, un fin dentro de su propia estructura, donde el sujeto se involucra de tal forma que permite la no distinción entre acción-pensamiento, pues se funden para dar origen a lo que se denomina experiencia óptima, experiencia caracterizada por el disfrute constructivo de lo que se hace y decidida a voluntad, un estado particular donde la experiencia del individuo fluye sin tener presentes las limitantes del medio o las experiencias pasadas, según Csikszentmihslyi y Csikszentmihslyi (1998): "esta unanimidad sugiere que el orden de la conciencia produce un estado experiencial muy específico, tan deseable que uno busca replicarlo con tanta frecuencia como le sea posible. A este estado hemos denominado flujo" (p. 43).

Para que una experiencia de flujo se dé, se requieren una serie de condiciones, estas son:

Los individuos deben establecer con un buen grado de precisión que pueden responder de manera adecuada a una tarea, es decir, el individuo establece qué hacer, sentirse con la capacidad de hacerlo y ejecutarlo. En palabras de Csikszentmihslyi y Csikszentmihslyi (1998), "la experiencia óptima requiere un equilibrio entre los desafíos percibidos en una situación determinada y las habilidades que la persona aporta a la misma" (p. 43). Además, la relación entre desafíohabilidades genera un proceso gradual en el que las actividades realizadas deben gradualmente aumentar la dificultad para mantener el flujo en los sujetos, evitando así el desinterés.

Las actividades generadoras de flujo son dispositivos que permiten al sujeto sentir la posibilidad de logro al alcance, retando al sujeto, pero dándole la posibilidad de demostrarse a sí mismo que puede con ello. De igual modo, una actividad debe de modo intencional permitir equilibrar el desafío y la habilidad para evitar sensaciones donde el sujeto inmerso en la actividad sienta que el reto lo sobrepasa y abandone la tarea, o el reto sea tan fácil que entre en procesos de aburrimiento y estrés.

Es necesario para una experiencia de flujo que los sujetos hayan desarrollado en el transcurso de sus interacciones una modelación de personalidad de característica autotélica, esto significa que el sujeto evalúa las condiciones a las cuales se enfrenta y establece la calidad del reto y la capacidad de sus habilidades para responder a él, tarea que requiere una gran habilidad de autodominio para asumir actividades solo en el espectro del equilibrio entre desafío y habilidad.

Csikszentmihslyi y Csikszentmihslyi (1998) proponen a su vez las siguientes dimensiones de la experiencia de flujo:

Las actividades deben disponer de metas claras y permitir de inmediato una retroalimentación concreta sobre el proceso de desarrollo de la actividad. El disfrute de una actividad, ya sea laboral, recreativa, de ocio o esparcimiento, está determinado por la posibilidad de reconocer el cumplimiento de metas parciales y generales por parte del individuo; cuando lo anterior no se da, la actividad no brinda la posibilidad de disfrute que necesita el flujo.

Cuando una actividad produce disfrute, logra hacer que el sujeto entre en estado de concentración, lo cual permite la unicidad entre la actividad y la generación de conocimiento. De acuerdo con los autores:

Como el flujo produce armonía en el self ${ }^{3}$, puede invertirse toda la atención en la actividad que se ejecuta en el momento. Esto produce la combinación de la actividad y el conocimiento, tan típico de las actividades placenteras. Uno carece simplemente de atención extra para pensar en alguna otra cosa (Csikszentmihslyi y Csikszentmihslyi, 1998, p.46).

\footnotetext{
3 Self hace referencia a la capacidad de los sujetos de dar cuenta de sí mismos, en la traducción literal se refiere al yo.
} 
La experiencia de flujo connota una alteración en la percepción del tiempo, esto significa que la experiencia no depende del tiempo, sino de la sensación que produce la actividad asociada con el disfrute y el placer, esto establece que el tiempo, como es comprendido, sufre una valoración contraintuitiva a la lógica común. Una hora de una actividad placentera y con sentido para el yo puede parecer un segundo o convertirse en un tiempo infinito donde se desea estar. La preocupación por el paso del tiempo como condición natural de la vida es contrarrestada por una experiencia llena de satisfacción y sentido de vida.

Lo anterior implica que una experiencia, si bien se da dentro de un determinado tiempo y espacio, no depende de un tiempo en particular para que la adquisición de significado alimente el yo, pasa por la conexión que se genera entre la situación y valoración emocognitiva ${ }^{4}$ como proceso de anticipación de una experiencia placentera, no pasa por establecer determinados tipos de tiempo-espacio para desarrollar actividades particulares, sino por una decisión consciente de alimentar la experiencia que se desea. Csikszentmihslyi y Csikszentmihslyi (1998) lo expresan así:

En el flujo, el self está en pleno funcionamiento, pero sin ser consciente de ser el ejecutor, y puede destinar toda la atención a la tarea que está ejecutando. En los niveles más retadores, las personas manifiestan experimentar una trascendencia del self, causada por la implicación anormalmente alta con un sistema de acción mucho más complejo del que encontramos en la vida cotidiana (p. 46).

Por último, Csikszentmihslyi y Csikszentmihslyi afirman que las actividades en las que se desarrolla la experiencia de flujo están por lo general fuera de las actividades rutinarias del trabajo, en la medida que la labor no se convierta en una acción retadora que cumpla con las condiciones y dimensiones del flujo. Por esta razón, las personas buscan otras actividades para entrar en estados de experiencia donde el descentramiento de lo cotidiano de sentido a la vida, es como el juego, un fin en sí mismo, que permite la recreación de la vida mental fuera de las condiciones y reglas que imponen los rituales diarios de la vida. La experiencia

4 Se refiere a la condición de monismo entre lo emocional y lo cognitivo, distinto al dualismo que diferencia los procesos mentales o psicológicos, en emocionales y cognitivos, como independientes y sin relación continua. óptima se busca en actividades de carácter no vocacional, rituales, de identidad social, de juego e interacción. Según los autores, "el flujo, en su mayor parte, se produce más en actividades no vocacionales que en las vocacionales" (p. 49).

Aporte de Kelly

Según Kelly (citado por Feixas, 2000), la experiencia obedece a un ciclo caracterizado por cinco fases:

- Anticipación de la experiencia. Se refiere a la intención que el sujeto posee sobre una determinada acción, una necesidad psicológica de esperar alguna retribución de una tarea emprendida asociada a esquemas establecidos por experiencias anteriores, en términos de Kelly, serían los constructos personales que guían la actividad humana.

- Implicación con el resultado. La experiencia solo se puede dar en el tiempo siempre y cuando el sujeto asuma de forma consciente implicarse en la acción de la experiencia para poder ver el resultado de las anticipaciones hechas.

- Encuentro con el acontecimiento. Una vez implicado el sujeto en una determinada experiencia, debe contrastar sus anticipaciones con el resultado y realizar un proceso de evaluación, donde haya:

- Validación/invalidación. De las anticipaciones hechas sobre las experiencias anteriores, es posible que se siga validando un determinado conjunto de experiencias con la consecuencia en ocasiones de no poder establecer con precisión si la experiencia favorece el desarrollo o crecimiento del sujeto en su vida interna o, por el contrario, que al invalidar las anticipaciones se abra paso a la reconfiguración de la experiencia y permita la:

- Revisión. De los diferentes factores asociados a la experiencia y con ello modificar la dirección de las anticipaciones a nuevos campos de aprendizaje y crecimiento personal. Luego de la revisión y modificación de las experiencias previas, surgen nuevas experiencias con anticipaciones nuevas, reiniciándose el proceso. 
La experiencia permite al individuo organizar los constructos sobre la vida, dirigirla hacia fines establecidos de acuerdo con los conocimientos y relaciones adquiridas en los procesos de interacción con los contextos donde se negocian símbolos y significados. En síntesis, se puede retomar de los argumentos expresados por los autores los siguientes aportes para ser relacionados con recreación:

\section{Dewey:}

- La experiencia es un medio a través del cual el sujeto se significa como individuo.

- La experiencia necesita de orientaciones pedagógicas $\mathrm{y}$, a su vez, cada experiencia es una mediación para otra experiencia.

- Toda experiencia se genera dentro de un contexto sociocultural.

\section{Glasersfeld:}

- La experiencia es una forma de conocimiento adaptativo.

- La experiencia se consolida a través de representaciones mentales y se comunica por medio de símbolos.

- La experiencia se adquiere en situaciones donde hay otros individuos con experiencia; la experiencia surge de la experiencia.

\section{Csikszentmihslyi y Csikszentmihslyi:}

- La experiencia es óptima cuando existe motivación intrínseca.

- Las experiencias significativas tienen fin en sí mismo.

- Una experiencia óptima no distingue entre acción y pensamiento.

\section{$\underline{\text { Kelly }}$}

- La experiencia se orienta como un encadenamiento de eventos que adquieren significado para el sujeto en su relación con el contexto dentro del cual interactúa, generando la necesidad de anticipar una situación en particular.

\section{RECREACIÓN Y SU RELACIÓN CON LA EXPERIENCIA (ANTECEDENTES)}

Al hacer una búsqueda de autores que escriben sobre recreación u ocio ${ }^{5}$ en relación con experiencia, encontramos a Cuenca (2004) que en sus postulados sobre el ocio reconoce la importancia de diferenciar la experiencia común (transitoria) de la experiencia verdadera (significativa), siendo una de las búsquedas del ocio y la recreación posibilitar la generación de experiencias que den al sujeto significado para su vida. Cuenca (2004) define la experiencia como:

\section{Un $<<$ acontecimiento vivido por una persona >> como consecuencia de $\ll$ haber sentido, conocido o presenciado algo $>>$, lo cual implica una acción di- recta del sujeto sobre su propia experiencia en la medida que es él quien determina la orientación de su propia experiencia" (p. 40).}

A su vez, establece como criterio de la experiencia su carácter subjetivo, en la medida que esta no se puede objetivar, no se pueden establecer parámetros de dirección, el sujeto inmerso dentro de una determinada situación se comporta de acuerdo con sus deseos, pasiones y necesidades. Cuenca (2004) lo explicita así: "La experiencia no se refiere habitualmente a un vivir pasivo, sino todo lo contrario, al estar despierto $\mathrm{y}$ activo que se relaciona con sentimientos, percepciones, pensamientos o deseos muy personales que resultan difíciles de objetivar" (p. 41).

Asimismo, Trilla (1993) reconoce la experiencia como una reconstrucción permanente del mundo social a través de las diferentes interacciones a las cuales se puede acceder en un contexto educativo; la experiencia se reconstruye tras una nueva experiencia ${ }^{6}$. En palabras de Trilla (1993), "cada experiencia educativa refuerza y facilita o contradice y niega a las otras" ( $p$. 86). Asimismo, Trilla considera que, dentro de la animación sociocultural (ASC), la experiencia no ha sido contemplada desde lo que pasa en el individuo, pues no se pregunta por la condición en la que se tiene que dar y como esta modifica al sujeto. En este sentido, Trilla (1993) expresa: "la ASC, por decirlo así, ha estado mucho más sociologizada que psicologizada. Proba-

5 Se enuncia ocio para hacer referencia a un concepto que normalmente se asocia a recreación. En el presente artículo, se retoma únicamente para indicar una relación entre la experiencia y conceptos semejantes.

6 Principio de la experiencia sugerido por Dewey. 
blemente este hecho puede explicarse por (...) que el sujeto de la ASC no es, en realidad, el individuo (...) sino el grupo, el colectivo o la comunidad" (p. 103).

Lo expresado por estos dos autores implica una relación entre recreación y experiencia, ya sea desde la postura donde se asume la experiencia como una condición de construcción psicológica y con perspectivas de desarrollo continuo o desde la postura sociológica de la animación sociocultural, que ve la experiencia como una condición dada en el entramado social y generadora de conocimiento a partir del conocimiento del otro. Estas dos perspectivas incorporan el proyecto de desarrollar en el(los) individuo(s) experiencias que den sentido y trascendencia a su vida mental e interacción social. De igual modo, coinciden en una acción de carácter pedagógico sobre la experiencia; es imperioso orientar esta por medio de acciones vinculantes entre sujetos o comunidades con formas de ser, y en algunos casos con fines sociales; esta apuesta pasa por ver la recreación y el ocio como una dimensión cultural más de lo humano, siendo digna de ser tomada en serio como un saber real y constituyente de relación sujeto-realidad.

Por último, es necesario hacer una distinción específica sobre la relación entre experiencia y recreación. Vista desde los argumentos anteriores, la experiencia puede generar la sensación de pertenecer de modo general a cualquier ámbito del ser humano, si es así, podría sugerirse la no relación entre estas dos; sin embargo, se apuesta por una experiencia recreativa, caracterizada por la diversión, el juego, lo lúdico, lo imaginativo, la fantasía, la interacción social, la di-versión de la realidad, lo festivo, la simulación, los sueños, entre otros. Apuesta que define un campo de intervención y saber, donde la recreación contribuye activamente a la significación de la realidad, el bienestar y calidad de vida de un individuo o de la sociedad.

\section{LA EXPERIENCIA COMO CATEGORÍA DE INTERVENCIÓN DE LA RECREACIÓN EN LA ESCUELA}

Uno de los ejes de trabajo de la recreación en la escuela puede orientarse a la generación de experiencias recreativas $^{7}$ significativas (Dewey), experiencias recreativas

7 Las cursivas hacen referencia a la proposición de la experiencia recreativa como unidad de análisis o categoría de intervención. adaptativas (Glasersfeld) o experiencias recreativas de flujo (Csikszentmihslyi y Csikszentmihslyi) a la construcción de posibilidades para dar sentido a la vida de los sujetos fuera de las condiciones rutinarias establecidas por el trabajo, la familia, el estudio, etc. Esta finalidad implica considerar la experiencia como eje central de cualquier tipo de intervención recreativa y hacer girar en torno a ella las intenciones formativas.

La experiencia, vista desde Dewey, aporta a la recreación la posibilidad de pensar que el sujeto decida implicarse dentro de su proceso de autoconstrucción de tal modo que pueda establecer el horizonte hacia el cual dirija su esfuerzos emocionales, siendo un fin la posibilidad de autoorganizar la vida mental hacia lugares donde el sujeto da significado a su acción en correspondencia con su contexto y sus intereses. La recreación debe generar la posibilidad de un conjunto de sensaciones donde el sujeto se permita experimentarse dueño de sus propios movimientos y deseos, donde las experiencias a las cuales se ve enfrentado modifiquen las ya existentes y posibiliten la creación de nuevas que le permitan crear nuevas formas de ethos individuales y culturales con los otros.

Dewey considera que la experiencia se puede dirigir a partir de un componente pedagógico hacia lugares intencionales, que pueden ser proporcionados desde fuera. Un buen conjunto de experiencias acumuladas permitirían al sujeto escoger aquellas orientadas al mejoramiento de la calidad de vida. En este sentido, la recreación posee la propiedad de retomar los lugares no comunes de la experiencia de los sujetos y logran a través de ellos la configuración de significados diferentes para la vida.

Asimismo, es necesario entender que la experiencia es una construcción individual derivada del entorno social, mediada por los objetos-medios dentro del alcance de la realidad dada por contextos específicos. En este sentido, la recreación puede generar contextos de realidad que permitan a los individuos una construcción diferente a la del entorno dentro del cual se encuentran, revalidar lo cotidiano en la vida de los individuos como una posibilidad transgresora sobre la hegemonía cognitivista de la escuela, logrando con ello la invención de mundos posibles alternos en los sujetos; esta característica de la recreación es una excusa para simular realidades dentro de las cuales se desee estar o aspirar a ellas de forma permanente. A su vez, sus posibilidades para construir realidades modifican la estructura de las representacio- 
nes mentales de los sujetos, configuran de formas alternas los símbolos culturales y, con ello, las capacidades de adaptación (Glasersfeld) a los contextos por los cuales transita la vida del yo.

De igual modo, al encadenar un conjunto de experiencias significativas para los individuos se logra crear mapas mentales de anticipación de la experiencia, lo que significa la búsqueda de sentido pleno de ser a través de lo experimentado; una consideración psicológica de querer estar en un determinado lugar y momento partiendo de los esquemas construidos por la interacción con situaciones que amplían el espectro de las representaciones que sobre nosotros y el entorno se construye. Un individuo se compromete más consigo mismo cuando encuentra en el contexto elementos que lo hacen filiarse sin obligarlo como condición de entrada, este proceso dentro de la escuela es posible por la dinamización de los espacios propuestos por la recreación, porque parte de la consideración de la interacción social como centro de su acción y no del individualismo lógico que pretende establecer contenidos cognitivos.

\section{PEDAGOGÍA Y RECREACIÓN}

Entrelazar la recreación a la escuela lía una diada que es atravesada por la pedagogía como elemento que le da cuerpo a esta relación. Aunque tratar de comprenderla implica asumir posturas teóricas, en tal caso, para entender desde donde se observa lo pedagógico, es necesario aclarar qué se entiende por ella.

Siguiendo a Zuluaga et al. (2003), se asume que la pedagogía es "la disciplina que conceptualiza, aplica y experimenta los conocimientos referentes a la enseñanza de los saberes específicos de la cultura". Esto lleva a asumir la pedagogía como un saber amplio no restringido a una acción instrumental, no aparece como una disciplina aplicada, sino como una disciplina constituyente que piensa y reflexiona sobre un proceso extensivo común a todo tipo de saber. Entonces podría decirse que lo pedagógico, en un primer momento, no pertenece solo al dominio de la escuela, sino a todo proceso, inclusive los no sistematizados. Dicho proceso ha de tener en cuenta a un alguien con un conocimiento susceptible de ser enseñado; un segundo momento corres- ponde a procesos de enseñanza donde se definen con cierta claridad unos determinados contenidos para ser enseñados a partir de una acción de enseñanza, y que en tal caso correspondería más a una institución como la escuela, en la medida que en ella se establecen fines teleológicos sobre la cultura y los individuos.

Es claro que el objeto de la pedagogía es la enseñanza de ciertos saberes de la cultura, en tal caso, la recreación se asume como uno de esos saberes de carácter social. Sin embargo, contiene un dominio de saber basado en un conjunto de interacciones sociales no definibles bajo premisas lógicas, susceptible de ser asumido como parte de la escuela en la medida que esta es un lugar de interacciones sociales donde parte de ellas pueden estar atravesadas por la recreación. A su vez, es necesario advertir que buena parte de los saberes a los que hace referencia la pedagogía poseen una estructura formal, esto enuncia un conjunto de saberes sistematizados y validados culturalmente como relevantes para el desarrollo social, por tanto, dignos de enseñanza, lo cual lleva inmersa una intención de formalidad lógica en la enseñanza, esto es, un alto contenido racional y cognitivo. En el caso de la recreación, el objetivo de su enseñanza no pasa por una formalidad lógica, sino por las posibilidades del desarrollo del pensamiento desde las conexiones que vinculan lo humano con lo humano, lo que representa un punto de partida diferente al enseñar, la recreación genera procesos de intersubjetivización, de ser con el otro.

Según Zuluaga et al. (2003), la enseñanza debe posibilitar un pensamiento "como habitando fuera de todo proceso preestablecido y conocido, de todo sistema concebido, de todo sujeto y de toda conciencia (...) el pensamiento sería entonces un pensamiento del afuera (...) lo impensado" (p. 210), esto se manifiesta como teleología de la pedagogía, la posibilidad de que, a través de ella, el individuo se relacione con aquello que no pertenece a lo cotidiano o normalizado, que asume direcciones por facilitar el espíritu creativo, la imaginación y el desdoblamiento de los esquemas tradicionales de la realidad para reconstruir nuevos ethos. Visión cercana a la apuesta de la recreación como transformadora de la realidad social, como activadora de ethos culturales basados en premisas no determinadas, sino construidas a varias manos. 
Lo anterior lleva implícitas preguntas en torno a contenidos, didácticas, metodologías de la recreación para ser objeto de enseñanza o reflexión de la pedagogía; sin embargo, es necesario establecer un marco de referencia puntual, la recreación no es una disciplina que contiene saberes definidos y sistematizados, pues su naturaleza no es la de contener conceptos o categorías explicativas de fenómenos a partir de la tradición iluminista; por el contrario, su saber es establecido por condiciones de relaciones culturales a partir de fenómenos lúdicos, creativos, imaginativos, que escapan al control de esquemas de saber-lógico y se manifiestan como expresiones colectivas o individuales hacia los otros, interactúa permanentemente con el entorno. Se manifiesta como una posibilidad de ser.

Esta postura de la recreación no la desconecta de la pedagogía como saber, por el contrario, la vincula como una estrategia a los procesos de desarrollo del individuo, contribuye a la formación, no desde lo lógico, sino desde lo dialógico, que se da en la interacción entre los individuos a partir de sus intereses, necesidades, gustos y satisfacciones. Asimismo, es necesario reconocer que lo pedagógico no pertenece exclusivamente a las acciones realizadas en una institución, en este caso escolar, sino que se encuentra en diferentes contextos en condiciones dispares y con intenciones diversas; aunque es evidente la existencia de principios reguladores de la educación ejercida en la escuela y determinan miradores sobre las posibilidades de la recreación como opción pedagógica, no es una limitante para poder pensar y construir modos de relación del saber recreativo al interior de la escuela. Las posibilidades presentes hoy en día a través de las mediaciones tecnológicas y las nuevas redes sociales cambian por completo la relación con el conocimiento cognitivo y en especial con el emocionar8, no es ya un problema de control sobre el saber, es una construcción con el otro de las realidades en las cuales desea vivir, y en este sentido la recreación tiene mucho que decir y aportar como saber no lineal.

Asimismo, la experiencia como unidad de análisis de la recreación se incorpora al sentido pedagógico, pasa a contenerse dentro de la enseñanza cuando se asume como susceptible de ser mediatizada, lo que significa que se pueden orientar experiencias a tra-

8 Concepto retomado de Humberto Maturana. vés de la enseñanza. En este caso, una experiencia del sujeto como ser trascendente que es la que propone la recreación. De igual modo, involucra un acto de conocimiento, la experiencia es acción sobre el entorno, es reconocimiento del ambiente, es contrastación de formas y sentires, no por medio de un orden preestablecido (deductivo), sino por descubrimiento, invención, sorpresa (por abducción e inducción); proceso que implica construcción de realidades a partir de ampliar teorías y no de comprobarlas, una forma de epistemología del encuentro. Esta posibilidad conforma un saber lúdico-creativo que permite al sujeto explorarse como dueño se sí y perteneciente a.

\section{CONCLUSIONES}

En primer lugar, asumir la recreación como fenómeno, hecho, objeto o saber de enseñanza implica una apuesta arriesgada al interior de la escuela; por un lado, porque no responde necesariamente a lógicas basadas en el control de contenidos académicos de forma seriada o análoga, por el contrario, se sustenta sobre la premisa de un saber dialógico presente de modo imperante en todas las formas de interacción social e incorporada por los individuos y comunidades de forma implícita en distintos contextos. Al pensar la recreación como una opción pedagógica, es evidente una reflexión sobre su finalidad en la escuela; si esta, en su seno alberga como una de sus actividades esenciales la enseñanza unida con la formación y el aprendizaje en sujetos que vivirán en el futuro, es menester incorporar a sus propósitos de enseñanza la flexibilidad en el saber por medio de la pregunta al otro sobre qué desea aprender o que se le enseñe. Hay grandes categorías del saber, es innegable, pero hay saberes que transitan de forma subterránea y poco lógica para la institucionalidad que dan en últimas el pegamento a los procesos de formación tan anhelados en la escuela, la recreación no direcciona experiencias a priori, las construye en lo cotidiano, en el momento, y las ata con las expectativas, el deseo, la fantasía y el escape al control.

En segundo lugar, el hecho pedagógico connota directamente una intencionalidad sobre los procesos educativos y formativos, dirige esfuerzos a desarrollar o posibilitar en los individuos y la sociedad ciertas acciones, comportamientos, intenciones y actitudes que permitan interactuar de forma armónica a partir de 
la diferencia. Las finalidades pedagógicas se orientan por construcciones y explicaciones teóricas (científicas o populares), en el presente caso, al incorporar la recreación a la tarea pedagógica, se hace necesaria la elaboración de categorías explicativas que permitan orientar la tarea al interior de la escuela, una de esas categorías es la experiencia como guía del proceso en recreación, una experiencia caracterizada por estar asociada al juego, la lúdica, lo festivo, los ritos, mitos, fantasía, y en especial con la posibilidad de experimentarse como sujeto en la felicidad. En este sentido, se es posible pensar la experiencia en los sujetos de las siguientes maneras:

1. El licenciado en recreación orienta parte de sus procesos pedagógicos a la generación de situaciones donde las características objetivas e internas sean parte del proceso formativo, esto quiere decir que el profesor de recreación, al mediar la experiencia recreativa del estudiante, tiene en cuenta las expectativas e intereses de estos para plantear el horizonte a seguir.

2. Las situaciones son mediaciones pedagógicas que posibilitan las experiencias de los sujetos, en este sentido, deben estar encadenadas para generar una continuidad experiencial a través de la interacción con otros sujetos o con objetos semióticos.

3. Al generar continuidad experiencial por medio de situaciones recreativas, se generan procesos de transformación de las representaciones mentales de los individuos y, con ello, se enriquece la vida mental y los procesos de aprendizaje social.

4. Toda situación plantea un reto al estudiante con el fin de movilizar sus emociones y motivaciones, este reto establece una correspondencia entre las habilidades del sujeto y la dificultad de este, permitiendo de modo general una valoración positiva de logro.

5. Toda situación planteada en el sentido de la continuidad experiencial pretende implicar al estudiante, lográndolo a partir de lo enunciado en el primer tópico.
En tercer lugar, se hace necesaria una revisión del concepto de recreación y sus relaciones con referentes teóricos y sociales; por un lado, se puede entender la recreación como un instrumento de control social (Herrera, 2013) ${ }^{9}$, erigido en relación opuesta a los medios de producción (trabajo), cuya función principal es la de diseñar el tiempo libre de los otros a partir de programas sociales de carácter institucional. Sin embargo, esta apreciación de carácter sociológico puede responder muy bien a ciertas características presentes en un tiempo socio-histórico muy relevante de mediados del siglo XX que hoy puede estar revalorada en función de las dinámicas derivadas de una sociedad más diversa, digital y con expectativas diferentes sobre su sentido. Retomar la recreación desde una postura pedagógica implica una profunda reflexión crítica sobre su función más extendida de control para dar lugar a miradas más contextuales y posibilitadoras de mundos posibles en los individuos. En este sentido, pensar la recreación desde categorías de intervención pedagógica permite asumir una mirada socio-crítica sobre la función de la recreación en un mundo contemporáneo donde lo inmediato es urgencia que ya pasó.

9 "Si bien la historia de la recreación está estrechamente vinculada con la institucionalización del tiempo libre, en la misma medida lo está respecto al trabajo, a la constitución de la democracia, a la paz, y, en general, a la formación de las identidades nacionales (...) la recreación emerge justificada desde discursos diversos que apuntan a su institucionalización y normalización especialmente para la infancia y la juventud, como recurso importante a la hora de educar para la producción" (Herrera, 2013, p. 43). 


\section{REFERENCIAS BIBLIOGRÁFICAS}

Cuenca, M. (2004). Pedagogía del ocio. Bilbao: Universidad de Deusto.

Csikszentmihslyi, M. y Csikszentmihslyi, I. (1998). Experiencia óptima: estudios psicológicos del flujo de la conciencia. Bilbao: Editora Descée De Brouwer.

Dewey, J. (1945). Educación y experiencia. Buenos Aires: Editorial Losada S.A.

Dewey, J. (2004). Democracia y educación. España: Ediciones Morata.

Feixas, G. (2000). Constructivismo y psicoterapia. Bilbao: Editora Descleé de Brouwer.

Glasersfeld, E. (1996). Aspectos del constructivismo radical. En M. Pakman (Comp.), Construcciones de la experiencia humana (pp. 23-50). Madrid: Editorial Gedisa.

Herrera, C. (2013). Apuntes para una historia de las relaciones entre juego y la recreación con el tiempo libre y el tiempo de ocio en Colombia a finales del siglo XX. Revista Lúdica Pedagógica, 2, (18), 35-48.

Trilla, J. (1993). Otras educaciones. Madrid: Anthropos.

Zuluaga, G., Echeverri, S., Martínez, A., Quiceno, H., Sáenz, J. y Álvarez, A. (2003). Pedagogía y epistemología. Bogotá: Magisterio. 